\title{
Reaction time as a function of the intensity and probability of occurrence of vibrotactile signals'
}

\author{
GEORGE A. GESCHEIDER, ${ }^{2}$ HAMILTON COLLEGE, JOHN H. WRIGHT, VIRGINIA POL YTECHNIC INSTITUTL: \\ BARRY J. WEBER, BRUCE M. KIRCHNER, AND ERIC A. MILLIGAN, HAMILTON COLLEGE
}

\begin{abstract}
Three Ss made judgments of the presence or abschce of a burst of 60 -cps libration on the index fingertip. The probability of $S$ 's reporting the presence of a signal was found to be influenced by signal probability and signal intensity. Mean reaction time for reporting the presence of a signal decreased as a function of signal intensity and signal probability whereas mean reaction time for reporting the absence of a signal increased as a function of signal intensity and signal probability. On trials where no signal was presented mean $R T$ for reporting a signal decreased with increases in the signal probubility whereas mean $R T$ for reporting the absence of a signal increased with increases in signal probability. The results were interpreted as support for the hypothesis that $S$ s decision time was longer the closer on the sensory continum a particular observation was to his criterion.
\end{abstract}

The empirical probabilities of S's reporting a signal on noise (N) trials and on signal-plus-noise (SN) trials comprise the most frequently employed measures of $S$ 's decision-making behavior in a signal-detection task. A second dimension of S'S behavior in the detection task. and one which has received relatively little experimental attention, is decision time. The Theory of Signal Detection (TSD) was originally constructed to account only for relationships between empirical response probabilities and certain parameters of the detection situation such as signal intensity, the costs and values associated with the various decision outcomes, and signal probability $[p(S)]$ (see Swets. Tanner, \& Birdsall, 1961). It would not be surprising, however, if the other dimension of detection behavior, decision time, were also related to these same parameters of the detection situation. If this were found to be the case, a strong argument could be made for the applicability of TSD to measures of reaction time (RT) in the detection of signals.

It has previously been proposed (Gescheider, Wright, \& Evans, 1968) that the relationships between RT and signal intensity and RT and $p(S)$ can be accounted for in terms of TSD if the assumption is made that RT becomes progressively longer the closer on the sensory continuum an observation is to the S's signal-present/signal-absent decision criterion. This assumption is consistent with the fact that disjunctive RT in a discrimination task increases as the psychological distance between stimuli decreases (Festinger, 1943; Kellogg, 1931). In the detection situation $\mathrm{S}$ is also confronted with a discrimination task since he must decide whether an observation has originated from a point on the sensory continuum above his criterion, and hence report "signal present," or from a point on the sensory continuum below his criterion, and hence report "signal absent." Consequently, the closer an observation is to the criterion the more difficult it should be, and the longer it should take, for $S$ to make his decisions.

Using vibrotactile signals, Gescheider et al (1968) examined the relationships between $\mathrm{RT}$ and signal intensity and RT and $\mathrm{p}(\mathrm{S})$ in the conventional signal-detection situation where the same signal intensity is employed within a single block of trials. In the conventional detection situation $S$ is informed at the beginning of a block of trials that a signal of constant intensity will be employed and, as a result, he is able to maximize the likelihood of correct detection decisions by locating his criterion on the sensation continuum at an optimal point relative to the $\mathrm{N}$ distribution and the $\mathrm{SN}$ distribution. During another block of trials in which a signal of different intensity is employed, $S$ can change the location of his criterion to maximize the likclihood of correct decisions based on observations originating from the $\mathrm{N}$ distribution and the new SN distribution. In a detection situation of this type Gescheider et al found (a) that with increasing $p(S)$ mean RT for reporting signals decreased and mean $\mathrm{RT}$ for reporting noise increased, and (b) that with increasing signal intensity mean RT decreased for correctly reporting both signals and noise. These findings were interpreted as support for the hypothesis that decision time is shorter for observations substantially above or below criterion than for observations close to criterion. The purpose of the present experiment was to gain further evidence concerning the general validity of this hypothesis by measuring RT in a more complex detection situation in which $S$ had to judge the presence or absence of signals of many different intensities presented in random order within a block of trials. It was assumed that in such a situation $S$ would not be able to employ different criteria for different signal intensities since there would be no way of knowing the intensity of the signal to be presented on a given trial. Consequently, in terms of TSD this situation involved (a) a single $\mathrm{N}$ distribution, the location of which remained fixed over an entire session, (b) an $\mathrm{SN}$ distribution for each signal intensity employed in the session, and (c) a single criterion. In this situation both RTs and response probabilities were obtained for correct and incorrect signal-present and signal-absent decisions. In half of the sessions $\mathrm{p}(\mathrm{S})$ was .25 , and in the remaining half of the sessions $\mathrm{p}(\mathrm{S})$ was .75.

\section{Subjects}

\section{METHOD}

The Ss were three male students at Hamilton College.

\section{Apparatus}

The signals were 60-cps sinusoidal vibrations applied for I sec to S's left index fingertip through a Goodmans V-47 vibrator. Signal intensity was regulated in $1-d B$ steps by a decade attenuator box. With his left index fingertip resting on a vibrator contactor $1 / 4$ in. in diam $S$ sat with his right hand in contact with a bidirectional telephone switch which could be closed to the left or right from its open position. A 1-sec white light served as a ready signal and was followed I to $2 \mathrm{sec}$ later by a $I-s e c$ observation interval which coincided with the 1 -sec presentation of a green light. The S's task was to judge the presence or absence of the tactile stimulus as quickly as possible following the onset of the green light. The $S$ made his judgment by closing the telephone switch to the right to indicate "signal present" or to the left to indicate "signal absent." The closing of the switch in either direction stopped a standard electric timer activated automatically at the onset of the observation interval. RTs were recorded to the nearest $5 \mathrm{msec}$. Knowledge of results was given to $\mathrm{S}$ after each judgment. 


\section{Procedure}

During a session signals with peak-to-peak amplitudes of -8 , $-6,-4,-2,0,2,4,6$, and $8 \mathrm{~dB}$ relative to $1.75 \mu$ were presented to $\mathrm{S}$ in random order. (The absolute threshold for each of the three Ss was measured prior to the beginning of the experiment by the method of limits. The mean absolute threshold was found to be $1.75 \mu$, and individual thresholds did not differ by more than $1 \mathrm{~dB}$ among the three Ss.) Over the 201 -h sessions in the experiment each $S$ made a total of 138 judgments for each of the nine signal amplitudes when $\mathrm{p}(\mathrm{S})$ was .75 and a total of 72 judgments for each signal amplitude when $p(S)$ was .25. The two values of signal probability, .25 and .75 , were counterbalanced over the 20 sessions, and $\mathrm{S}$ was told the value of $\mathrm{p}(\mathrm{S})$ at the beginning of each session. The $\mathrm{N}$ and $\mathrm{SN}$ trials occurred in random order for each $\mathrm{S}$ in each session. Prior to the beginning of the experiment Ss were given six 1-h practice sessions using the above procedure with the exception that $\mathrm{p}(\mathrm{S})$ was .50 . After about four sessions RT performance ceased to improve with further practice.

\section{RESULTS AND DISCUSSION}

It is assumed in TSD that the area under a particular SN distribution above criterion corresponds to the experimentally obtained proportion of correct "yes" decisions on the signal-present trials for that signal amplitude, and that the area under the $\mathrm{SN}$ distribution below criterion corresponds to the experimentally obtained proportion of incorrect "no" decisions on the signal-present trials for that signal amplitude. Similarly, it is assumed that the empirical proportions of incorrect "yes" and correct "no" decisions on the signal-absent trials correspond to the areas under the $\mathrm{N}$ distribution located above and below criterion, respectively. Thus, it was possible to estimate the location of the mean of the $\mathrm{N}$ distribution with respect to the criterion by converting the proportion of "yes" responses obtained on trials where the

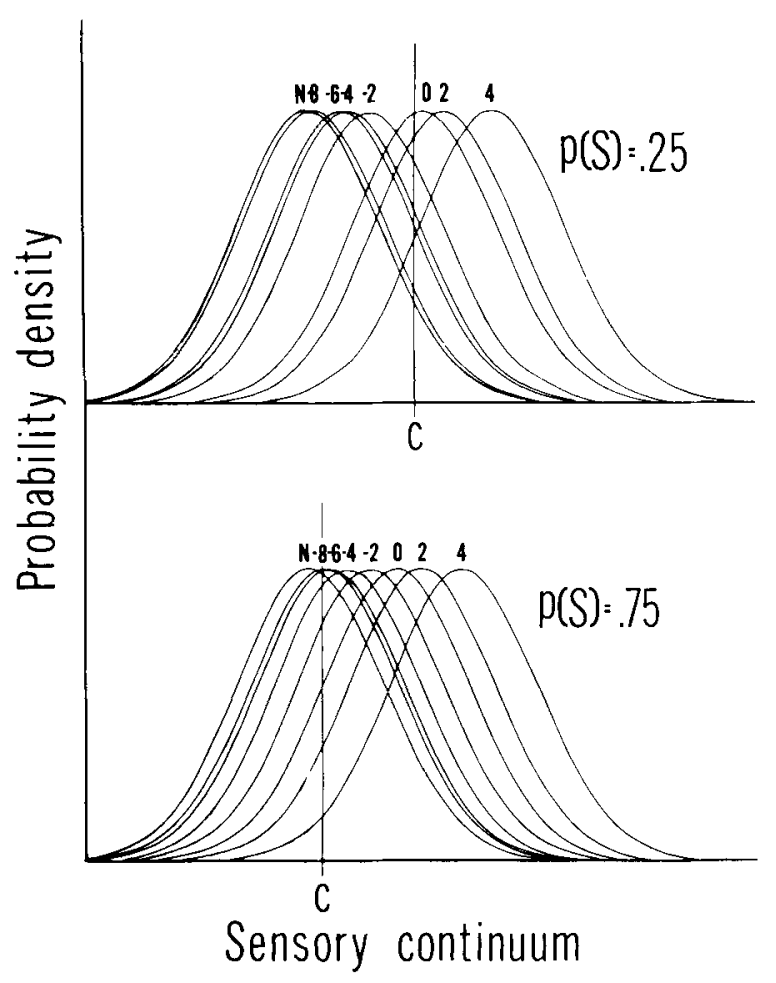

Fig. 1. Estimations of $N$ and $S N$ distributions relative to $S$ 's criterion for $p(S)$ values of .25 and .75 .

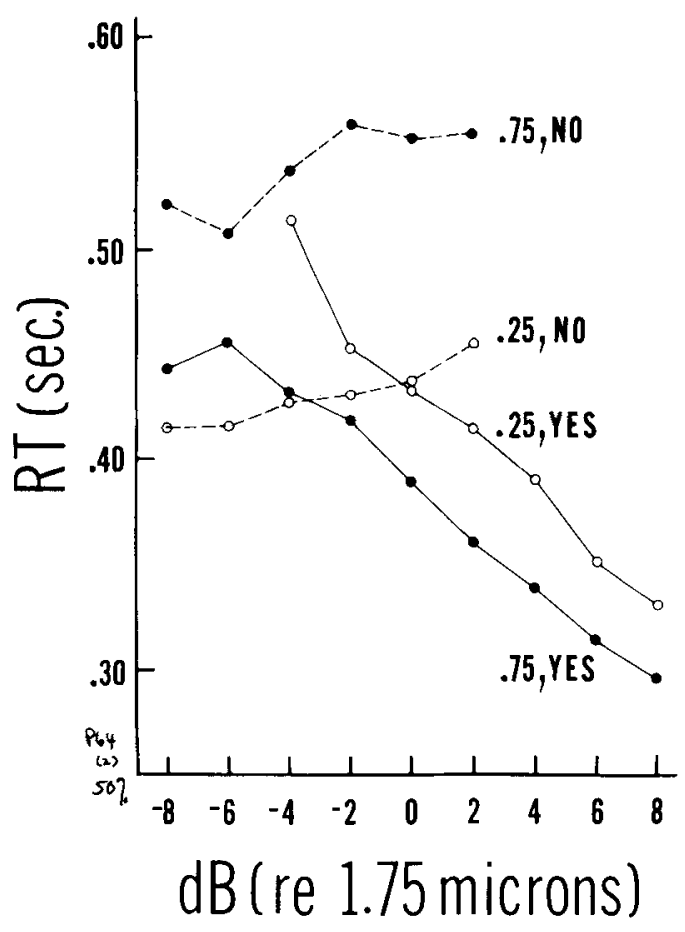

Fig. 2. Mean RT (in seconds) for signal-present and for signal-absent judgments obtained under $\mathrm{p}(\mathrm{S})$ values of .25 and .75 plotted as a function of signal intensity.

signal was absent into $z$ scores. Likewise, the location of the mean of an SN distribution relative to the criterion was estimated by converting into a $z$ score the proportions of "yes" responses obtained on trials in which signals of a particular amplitude were presented. In addition, the assumption that the $\mathrm{N}$ and $\mathrm{SN}$ distributions are normal in form, coupled with the further assumption that all $\mathrm{N}$ and $\mathrm{SN}$ judgments in a session were based upon a single criterion, permitted estimation of the relative distances on the sensory continuum among the means of the SN distributions under each of the $\mathrm{p}(\mathrm{S})$ conditions.

Estimations of the $\mathrm{N}$ distribution and the SN distributions generated by several of the signal amplitudes employed in the experiment are presented in Fig. 1 for $p(S)$ values of .25 and .75 to illustrate the location of each of these distributions with respect to $\mathrm{S}$ 's decision criterion. The mean of the $\mathrm{N}$ distribution was arbitrarily assigned the same value on the sensory continuum for both the .25 and $.75 \mathrm{p}(\mathrm{S})$ conditions. Since there were considerably more $\mathrm{N}$ observations than $\mathrm{SN}$ observations for any single signal amplitude, the means of the $\mathrm{N}$ distributions under the two $\mathrm{p}(\mathrm{S})$ values, rather than the means of the .25 and $.75 \mathrm{SN}$ distributions for a particular signal amplitude, were given the same value on the sensory continuum. Theoretically, the two SN distributions for a particular signal amplitude should be located at the identical place on the sensation magnitude continuum. Generally there was fairly close correspondence between the locations of the SN distributions for a particular signal intensity obtained under the two $p(S)$ conditions. As was the case when $S$ was required to detect a vibrotactile signal of one particular intensity during a block of trials (Gescheider et al, 1968), increasing the value of $p(S)$ resulted in a lowering of $S$ 's decision criterion.

Mean RTs as a function of signal amplitude for signal probabilities of .25 and .75 are presented in Fig. 2. The solid and dashed curves are plots of mean RT on SN trials for signal-present and signal-absent decisions, respectively. Mean RTs are not presented in Fig. 2 for correct "yes" decisions for 
Table 1

Mean RT (in Sec) for Signal-Present and Signal-Absent Judgments Obtained under Two $\mathbf{p}(\mathbf{S})$ Conditions

\begin{tabular}{lll}
\hline & \multicolumn{3}{c}{$\mathrm{p}(\mathrm{S})$} \\
\cline { 2 - 3 } & .25 & .75 \\
\hline Yes & .482 & .431 \\
No & .414 & .532 \\
\hline
\end{tabular}

signals weaker than $-4 \mathrm{~dB}$ under $\mathrm{p}(\mathrm{S}) .25$, or for incorrect "no" decisions for signals stronger than $2 \mathrm{~dB}$ under either 25 or $.75 \mathrm{p}(\mathrm{S})$. In none of these cases was there a sufficient number of decision outcomes ( 30 or more) to yield a reliable estimate of mean RT. It is readily apparent from inspection of Fig. 2 that increases in signal amplitude had the effect of decreasing mean RT for correct "yes" decisions and increasing mean RT for incorrect "no" decisions. These findings strongly support the hypothesis that signal-present/signal-absent decisions for sensation magnitudes near criterion take longer than for sensation magnitudes substantially above or below criterion. The weakest signals produced observations that were substantially below criterion, and hence resulted in more rapid "no" decisions, while the strongest signals produced observations that were substantially above criterion, and hence resulted in more rapid "yes" decisions. The signal amplitudes producing sensation magnitudes close to criterion resulted in greater RTs for both "yes" and "no" decisions. Signal strength appears to have a somewhat greater effect on RT for correct "yes" decisions than on RT for incorrect "no" decisions. Presumably increasing signal strength resulted in lower mean RTs of "yes" decisions by decreasing neural transmission time and by increasing the proportion of observations that were substantially above criterion. If increasing signal strength increased the proportion of the SN distribution substantially above criterion it must have also decreased the proportion of the SN distribution substantially below criterion, which accounts for the observed increase in mean RTs for "no" decisions as a function of signal strength. The decrease in neural transmission time with increasing signal strength, however, may have tended to reduce the degree to which mean RT for "no" decisions increased with signal strength. At least there is no way that changes in neural transmission time could augment the effect of signal strength as was the case with mean RT for "yes" decisions.

Mean RT was also affected by $p(S)$ on both $N$ and SN trials (Fig. 2 and Table 1). Fig. 2 reveals that mean RTs on SN trials were uniformly higher for incorrect "no" decisions under $\mathrm{p}(\mathrm{S})$ .75 than for incorrect "no" decisions under $p(S) .25$ but were uniformly lower for correct "yes" decisions under $p(S) .75$ than for correct "yes" decisions under $p(S) .25$. On $N$ trials mean RT for incorrect "yes" decisions was lower under $p(S)$ .75 than under $\mathrm{p}(\mathrm{S}) .25$ and mean RT for correct "no" decisions was higher under $p(S) .75$ than under $p(S) .25$ (Table 1). These results agree with those of Carterette,
Friedman, and Cosmides (1965), and Gescheider et al (1968) who found RT for "yes" decisions to decrease with increasing $\mathrm{p}(\mathrm{S})$ and RT for "no" decisions to increase with increasing $p(S)$ in a conventional signal-detection situation where the same signal intensity is employed throughout a single block of trials. Thus, in both $\mathrm{N}$ and $\mathrm{SN}$ situations it appears that $\mathrm{S}$ will tend to make more rapid "yes" decisions and less rapid "no" decisions when $\mathrm{p}(\mathrm{S})$ is high (e.g., .75) and his criterion is low than when $p(S)$ is low (e.g., .25) and his criterion is high. As the criterion is lowered, larger portions of the $\mathrm{N}$ and $\mathrm{SN}$ distributions become located substantially above the criterion, reducing mean RT for "yes" decisions. However, when the criterion is lowered by increasing $\mathrm{p}(\mathrm{S})$, smaller portions of the $\mathrm{N}$ and SN distributions remain substantially below criterion, and this causes the RTs for "no" decisions to increase as a function of $\mathrm{p}(\mathrm{S})$ in both the $\mathrm{N}$ and $\mathrm{SN}$ situations.

The results of the present experiment, in which $S$ using a single criterion judged the presence or absence of signals of varying strengths presented randomly within a single block of trials, provide further support for the applicability of TSD to measures of RT in detection situations. The effects of signal intensity and $p(S)$ on RT can be accounted for in terms of TSD when the assumption is made that the closer on the sensory continuum an observation is to the criterion by which $S$ makes his decisions the longer his decision times will be.

\section{REFERENCES}

CARTERETTE, E. C., FRIEDMAN, M. P., \& COSMIDES, $R$ Reaction-time distributions in the detection of weak signajs in noise. Journal of the Acoustical Society of America, 1965, 38, 531-542.

FESTINGER, L. Studies in decision: II. An empirical test of a quantitative theory of decision. Joumal of Experimental Psychology, $1943,32,411-423$.

GESCHEIDER, G. A., WRIGHT, J. H., \& EVANS, M. B. Reaction time in the detection of vibrotactile signals. Journal of Experimental Psychology, 1968, 77, 501-504.

KELLOGG, $W$. N. The time of judgment in psychometric measurement. American Journal of Psychology, 1931, 43, 65-86.

SWETS, J. A., TANNER, W. P., JR., \& BIRDSALL, T. G. Decision processes in perception. Psychological Review, 1961, 68, 301-340.

\section{NOTE}

1. This invistigation was supported by Public Health Service research grant NB 07620 from the National Institute of Neurological Diseases and Blindness.

2. Address: Department of Psychology, Hamilton College, Clinton, $\mathrm{N}, \mathrm{Y}$.

(Accepted for publication September 11, 1968.) 